\title{
Tsafon
}

Revue d'études juives du Nord

$72 \mid 2016$

Juifs, Israéliens, dans la littérature française et israélienne

\section{L'obsession de la dualité chez Naïm Kattan}

\section{Éric Hocquette}

\section{(2) OpenEdition \\ Journals}

Édition électronique

URL : https://journals.openedition.org/tsafon/364

DOI : $10.4000 /$ tsafon.364

ISSN : 2609-6420

Éditeur

Association Jean-Marie Delmaire

Édition imprimée

Date de publication : 1 décembre 2016

Pagination : 89-113

ISSN : $1149-6630$

\section{Référence électronique}

Éric Hocquette, «L'obsession de la dualité chez Naïm Kattan », Tsafon [En ligne], 72 | 2016, mis en ligne le 31 mai 2018, consulté le 24 juin 2021. URL : http://journals.openedition.org/tsafon/364 ; DOI : https://doi.org/10.4000/tsafon.364 


\title{
L'obsession de la dualité chez Naïm Kattan
}

\author{
Éric Hocquette*
}

Auteur quasiment inconnu du grand public français, si ce n'est à travers son roman autobiographique Adieu Babylone ${ }^{1}$, Naïm Kattan est au contraire un écrivain célèbre, connu et reconnu au Canada, son pays d'adoption. Issu d'une famille juive modeste, il est né en 1928 à Bagdad, et se tourne très jeune vers la littérature. Faisant fi des diktats culturels, il s'intéresse à toutes les littératures, tant arabe qu'occidentale; des ouvrages tels que Les Nourritures terrestres d'André Gide trouvent tout naturellement leur place dans sa bibliothèque, à l'instar des poèmes des grands auteurs classiques arabes comme Al Zahawi ${ }^{2}, \mathrm{Al} \mathrm{Marri}^{3}$ ou Al Moutannabbi ${ }^{4}$. Au début de sa carrière, il s'intéresse au mouvement surréaliste qu'il introduit en Irak par le biais d'articles qu'il rédige dans différentes revues littéraires (Al majalla, Al Fikr al hadith ou Al Waqt al dhaie). Mais c'est véritablement sa rencontre avec la littérature française qui transforme sa vie, tant sur le plan personnel que professionnel. Son amour pour la langue de Molière lui ouvre les portes de la France : après avoir obtenu une bourse d'études de la part du gouvernement français, il prend la direction de Paris pour aller étudier à la Sorbonne entre 1947 et

\footnotetext{
* Doctorant, Université Charles de Gaulle - Lille 3.

${ }^{1}$ Naïm Kattan, Adieu Babylone, mémoires d'un Juif d'Irak, Paris, Albin Michel, 2003, 307 p., première édition en 1975.

2 Jamil Sidqi Al Zahawi (1863-1936) : poète et philosophe irakien, considéré comme l'un des plus grands poètes du monde arabe, défenseur des droits de la femme.

3 Abul Ala Al Marri (973-1057): poète syrien connu pour sa virtuosité et pour l'originalité et le pessimisme de sa vision du monde. Ses poèmes sont construits sur la base d'une tristesse existentielle profonde, faisant du pessimisme une ligne de conduite et le départ de toute réflexion philosophique.

${ }^{4}$ Al Moutannabbi (915-965) : poète irakien, on considère que ses 326 poèmes sont une représentation de sa vie. Il commença à écrire à l'âge de 9 ans. Ses thèmes de prédilection sont le courage, la philosophie de vie et la description des batailles.
} 
1951. Ne trouvant pas sa place en France, ni en Europe, Naïm Kattan décide dès lors de partir. Son fort attachement à la langue française l'amène tout naturellement à prendre la direction du Canada en 1954, pays dans lequel il s'installe définitivement, sans pour autant couper les ponts avec le français.

Auteur prolifique, Naïm Kattan a publié près d'une quarantaine d'ouvrages de différents types (romans, nouvelles, essais, pièces de théâtre). Il est distingué à plusieurs reprises par ses pairs que ce soit au Canada ou à l'étranger : officier de l'Ordre du Canada en 1983, chevalier de l'Ordre national du Québec en 1990, légion d'honneur en 2002, officier de l'ordre des Arts et des Lettres de France. Il occupe également de hautes fonctions dans le domaine de la culture au Canada et profite de cette position pour mettre en valeur les lettres canadiennes, qu'elles soient francophones ou anglophones.

En 1970, il publie son premier essai sous le titre: Le Réel et le théâtral ${ }^{5}$. Suite à cet essai, que l'on peut qualifier de point de départ de la pensée kattanienne, l'intellectuel irako-canadien poursuit et étoffe sa vision du monde à travers ses écrits, fictifs ou non. Tel un sociologue, Kattan passe en revue l'être humain sous tous les angles, que ce soit à travers ses relations aux autres, son rapport à la vie ou avec le monde. Mais ce qui frappe lorsque l'on commence à lire et à analyser les différents ouvrages de son œuvre, c'est cette volonté de la part de l'auteur de mettre constamment deux personnes, deux notions en confrontation, ou d'aborder des concepts qui incluent déjà une idée de confrontation entre deux notions. Tout ce qui est duel attire Naïm Kattan, à tel point que la dualité apparaît dès lors comme une obsession chez l'auteur. Cette obsession, consciente ou non, pour la dualité ne se cantonne pas uniquement à la mise en "confrontation» dans ses différents écrits d'éléments présents dans l'intrigue de ses fictions, ou bien de concepts dans ses essais, mais elle se retrouve également, comme nous allons le voir, dans la structure, la construction même de ses écrits.

\section{La dualité dans la construction du livre}

En faisant l'analyse des titres des différents écrits de Naïm Kattan, on constate que la plupart des titres de ses essais sont formés sur le même schéma : l'auteur utilise les deux termes des notions qu'il va aborder, puis

${ }^{5}$ Le Réel et le théâtral, Montréal, Bibliothèque Québécoise, 2005, 201 p., première édition en 1970 (Montréal, éditions Hurtubise HMH). 
il les met en relation par le biais de la conjonction de coordination « et » (parfois « ou ») : La Mémoire et la promesse ${ }^{6}$, Le Désir et le pouvoir ${ }^{7}$, La Parole et le lieu ${ }^{8}$, Culture : alibi ou liberté ? ${ }^{9}$, Le Réel et le théâtral ${ }^{10} \ldots$ On retrouve ce même schéma pour certains titres de chapitres comme dans La Mémoire et la promesse, essai qui est lui-même découpé en deux parties: Les nomades et les errants, Le lieu et l'espace, La culture et l'État, ou encore L'homme et l'œuvre.

La mise en parallèle, en confrontation des éléments sous cette forme duelle ne se résume pas aux titres des ouvrages ou des chapitres ; Naïm Kattan pousse la "dualité structurelle » encore plus loin dans la construction même de ses ouvrages en l'explorant, l'exploitant sous différentes formes. Dans le roman Le gardien de mon frère ${ }^{11}$, qui relate la relation tumultueuse entre deux frères (Raphaël et Gabriel) que tout semble opposer, l'intrigue ne se déroule pas en suivant un schéma structurel construit sur une succession d'événements respectant la chronologie. Naïm Kattan a volontairement découpé ce roman en faisant alterner les pensées de deux principaux protagonistes sur des événements précis, des expériences de vie qu'ils ont en commun. Grâce à ce découpage alternatif, l'auteur met en perspective les pensées des deux personnages qui peuvent ainsi donner leurs propres visions, leurs propres versions sur des faits présents ou passés. Ce procédé permet également aux deux frères, dont la relation est au plus mal, de se répondre d'une certaine façon l'un à l'autre, le lecteur devenant dès lors le thérapeute écoutant et arbitrant ce couple en crise.

Naïm Kattan avait déjà utilisé un tel procédé d'écriture, mais de façon plus «parcellaire», dans un autre de ses romans: L'Amour reconnu ${ }^{12}$.

L'auteur utilise à nouveau ce même procédé «d'alternance structurelle » dans un autre de ses romans, mais sous une autre forme. Dans Le Veilleur ${ }^{13}$, Éliahou, rabbin dans la ville de Montréal, tente de trouver des solutions aux problèmes des membres de sa communauté, de

\footnotetext{
${ }^{6}$ Naïm Kattan, La Mémoire et la promesse, Paris, Denoël, 1979, 160 p.

${ }^{7}$ Naïm Kattan, Le Désir et le pouvoir, Montréal, Éditions Hurtubise, 2005, 209 p.

${ }^{8}$ Naïm Kattan, La Parole et le lieu, Montréal, Éditions Hurtubise, 2004, 307 p.

${ }^{9}$ Naïm Kattan, Culture : alibi ou liberté ?, Montréal, Éditions Hurtubise, 1996, 150 p.

${ }^{10}$ Naïm Kattan, Le Réel et le théâtral, Montréal, Bibliothèque Québécoise, 2005, 201 p., première édition en 1970 (Montréal, Éditions Hurtubise HMH).

${ }^{11}$ Naïm Kattan, Le Gardien de mon frère, Paris, Éditions du Rocher, 2004, 187 p.

12 Naïm Kattan, L'Amour reconnu, Montréal, Éditions de l'Hexagone, 1999, 188 p.

${ }^{13}$ Naïm Kattan, Le Veilleur, Montréal, Éditions Hurtubise, 2009, 260 p.
} 
répondre à leurs interrogations. C'est au cours de ses entretiens avec les différents membres de sa congrégation que le personnage principal se remémore sa jeunesse à New-York, sa rencontre avec Emma sa future femme, son voyage avec cette dernière en Israël, l'influence de Abe sur sa vocation de rabbin. L'intrigue de cet ouvrage se construit donc sur une alternance, une dualité présent/passé. Mais contrairement au roman Le Gardien de mon frère, l'alternance entre le passé et le présent se fait à l'intérieur même des chapitres qui sont découpés par des flash-back brutaux, sans lien évident ou explicite avec la situation présente. On retrouve dans d'autres romans de Kattan l'utilisation de flash-back à l'image du Veilleur comme dans le roman L'Anniversaire ${ }^{14}$, ou encore dans le roman Farida ${ }^{15}$ même si dans ce dernier elle est présente sous une forme plus légère.

Dans La Célébration ${ }^{16}$, Naïm Kattan expérimente une nouvelle mise en perspective physique de la dualité sous une forme différente par rapport à ce que nous venons de voir. Ici, le livre se divise en deux parties qui correspondent à deux moments importants de la vie de PierreNathan, le personnage principal de ce roman. La première partie relate la (re)découverte par Pierre-Nathan de sa judéité, une (re)découverte qui le fait rencontrer Dina, une jeune femme juive mariée et mère d'un jeune enfant, avec laquelle il a une aventure. Cette liaison est destructrice dans la mesure où Pierre-Nathan finit par divorcer de son épouse Christine, et surtout, le plonge dans un état de mal-être qui lui fait perdre pied et le pousse à tout quitter pour tenter de se retrouver, se reconstruire. La deuxième partie voit la renaissance de Pierre-Nathan, une renaissance qui devient possible grâce à sa rencontre avec Tova. Cette dernière lui permet de reprendre goût à la vie, de retrouver confiance en lui, de revivre.

\section{Les thèmes de la dualité dans la pensée kattanienne}

\section{L'Amérique et le Brésil}

Tout ce qui est duel fascine l'intellectuel irako-canadien, une fascination qui explique le nombre important de thèmes, de notions, ou de situations impliquant ou mettant en scène une mise en parallèle,

\footnotetext{
${ }^{14}$ Naïm Kattan, L'Anniversaire, Montréal, Éditions Québec Amérique, 2000, 164 p.

15 Naïm Kattan, Farida, Montréal, Guernica Editions Inc, 2015, 248 p. (anglais première édition en français en 1991).

${ }^{16}$ Naïm Kattan, La Célébration, Sherbrooke, Éditions de l'Hexagone, 1997, 204 p.
} 
présents dans les différents écrits de l'auteur. L'un des premiers thèmes porteur de cette dualité, selon l'auteur, se trouve dans la figure du continent américain au sens large du thème. En effet, on constate que le continent américain occupe une place importante dans l'univers kattanien en tant que lieu. Que ce soit à travers ses travaux et/ou articles, ou bien à travers ses fictions, l'action se situe essentiellement au Canada, et plus généralement sur l'ensemble du continent américain (États-Unis, Brésil, Argentine). Il existe un lien particulier entre l'enfant de Bagdad et le Nouveau Monde, et en particulier pour son Canada d'adoption. On pourrait même qualifier ce lien de relation amoureuse et ajouter que la fameuse fiancée promise de son dernier roman autobiographique ne serait en fait que cette Amérique qu'il chérit tant ${ }^{17}$. Cette relation particulière avec le continent américain s'explique, entre autres, par le fait que Naïm Kattan ne s'est pas senti dépaysé lorsqu'il est arrivé pour la première fois aux États-Unis. Au contraire, il a trouvé une certaine familiarité sur ce continent qui lui était jusque-là complètement inconnue :

Ici, en Amérique, je suis chez moi, je renoue avec l'espace sans bornes. Un sable invisible recouvre perpétuellement le travail des hommes, efface leur entreprise toujours recommencée de marquer la surface de la terre, annonçant leur venue, affirmant leur passage, victimes de leur vanité. ${ }^{18}$

Les villes américaines sont, selon lui, également très ressemblantes aux villes orientales : « rien ne ressemble plus à une ville du MoyenOrient qu'une ville d'Amérique $»{ }^{19}$.

Même si le Canada apparaît comme le lieu de prédilection dans lequel se déroulent les intrigues kattaniennes, le Brésil occupe lui aussi une place tout aussi importante dans la pensée de l'auteur. En effet, le pays apparaît souvent en toile de fond dans nombre de ses romans et nouvelles; beaucoup de personnages de l'univers kattanien s'y rendent comme Ezra dans La Fortune du passager ${ }^{20}$ ou Edgar dans Le Réveil des distraits $^{21}$. D'autres encore l'évoquent à travers leurs souvenirs comme René Shems dans L'Anniversaire. À noter que l'auteur fait également souvent référence au Brésil dans ses essais ou ses articles, qu'il aime à le prendre en exemple pour illustrer quelques points de sa pensée. Plus

\footnotetext{
${ }^{17}$ Naïm Kattan, La Fiancée promise, Montréal, Éditions Hurtubise, 1983, 231 p.

${ }^{18}$ Naïm Kattan, La Mémoire et la promesse, p. 14.

${ }^{19}$ Naïm Kattan, L'Autre Amérique dans La Parole et le lieu, p. 98.

${ }^{20}$ Naïm Kattan, La Fortune du passager, Montréal, Éditions Hurtubise, 1989, 343 p.

${ }^{21}$ Naïm Kattan, Le Réveil des distraits, Montréal, Éditions Hurtubise, 2012, 299 p.
} 
encore que les Nord-Américains, Naïm Kattan compare souvent, de façon directe ou indirecte, le peuple brésilien au peuple irakien. Une comparaison que l'on retrouve parfois dans la bouche de certains de ses personnages, comme avec Ezra qui est d'origine irakienne dans $L a$ Fortune du passager :

Il était sensible à la présence des hommes et des femmes, de l'existence des rues et des voitures qui sillonnaient. Sons familiers, démarches reconnues malgré l'étrangeté de surface. Il était chez lui, comme s'il avait regagné son foyer, retrouvé la chaleur du sein maternel [...] Ezra était déjà épris de cette ville. Ce n'était pas que l'alliance de la montagne et de la mer, le grouillement humain, non plus que l'air chaud et le soleil radieux, mais le rappel d'un passé subitement purifié de ses scories, d'une mémoire purifiée des souvenirs malheureux. Il était libre de toute peur, épargné de toute hostilité de la part des policiers et des représentants de l'autorité. ${ }^{22}$

Mais quel est le lien entre l'Amérique et l'obsession de la dualité chez Naïm Kattan ? Selon l'auteur, l'Amérique est pénétrée de la dualité, que ce soit dans sa composition actuelle, ou bien à travers son histoire. Dans La Mémoire et la promesse, Kattan affirme que depuis sa découverte par les Européens, le continent américain est traversé par une « dualité construite» par les différents acteurs de son Histoire. Que ce soit au nord ou au sud, on se retrouve toujours en présence d'une dualité, d'une confrontation: autochtones/colons, entre les colons eux-mêmes (dualité Français/Anglais au nord, et au sud Espagnols/Portugais), nord anglo-saxon/sud latino. Cette dernière va même jusqu'à se ressentir notamment à travers la poésie comme le souligne Naïm Kattan dans son essai sur les écrivains d'Amérique latine dans les chapitres consacrés à Octavio $\mathrm{Paz}^{23}$ ou sur Reinaldo Arenas ${ }^{24}$.

Une autre forme de dualité, présente dans l'espace américain selon la pensée kattanienne, est le parallèle que fait l'auteur entre l'Amérique et Israël. Pour l'intellectuel irako-canadien, l'Amérique apparaît comme étant une nouvelle terre promise, ou plus exactement, une terre de promesses, une terre permettant une possible concrétisation de la parole

\footnotetext{
${ }^{22}$ Naïm Kattan, La Fortune du passager, pp. 129-131.

${ }^{23}$ Octavio Paz (1914-1998) : écrivain mexicain, lauréat du Prix Nobel de littérature en 1990.

${ }^{24}$ Reinaldo Arenas (1943-1990) : écrivain cubain persécuté par le régime castriste pour son homosexualité, il est surtout connu pour son roman autobiographique Avant la nuit qui fut porté au cinéma par le réalisateur américain Julian Schnabel en 2000 qui reçut le Grand Prix spécial du jury à la Mostra de Venise la même année.
} 
divine : « L'aventure américaine m'est apparue alors comme une tentative consciente, délibérée, de concrétiser une parabole biblique. L'appel au départ, l'attente, le désert, l'exil et l'investissement d'une terre promise $»^{25}$. Le parallèle va jusqu'à associer l'Amérique à l'épisode biblique qui suit la sortie du peuple juif d'Égypte ${ }^{26}$. Même si l'auteur affirme dans la suite de son raisonnement que la promesse ne sera jamais tenue, à cause notamment de l'attitude des hommes ou à cause de leur relation avec le «réel», le parallèle entre ces deux territoires reste fortement ancré dans la pensée kattanienne.

\section{Le masculin et le féminin}

Les romans et nouvelles de Naïm Kattan décrivent dans leur très grande majorité des histoires d'amour, de sexe, voire très souvent des histoires extraconjugales. Dans la littérature kattanienne, les couples sont rarement décrits comme fidèles et peu de personnages ont connu au cours de leur vie un(e) seul(e) partenaire. La très grande majorité d'entre eux sont dans une logique de "consommation sexuelle à l'extrême " comme Gabriel dans Le Gardien de mon frère, ou bien entretiennent des relations adultérines comme Pierre-Nathan dans La Célébration. Cette consommation excessive apparaît plus, pour les personnages de l'univers kattanien, comme un recours, un exutoire leur permettant de fuir, d'échapper à un présent et/ou un passé lourd à gérer, ou de combler un manque, apaiser une solitude. Tous les personnages qui sont dans cette situation de consommation sexuelle poussée à l'extrême sont dans une position personnelle, familiale, sociale ou identitaire précaire. Ils sont tous d'une certaine façon dans un exil psychologique.

Il existe dans la pensée kattanienne un lien particulier de proximité entre sexualité et exil. Chez Naïm Kattan, le sexe apparaît pour les personnages comme le moyen d'échapper à cette situation d'inconfort social ou identitaire. Mais en réalité le sexe ne permet pas de briser cette solitude, d'apaiser cette souffrance chez un individu. Il n'apparait pas comme étant une réponse adéquate. Pire, il semble même parfois amplifier cet inconfort, cette angoisse, cette solitude chez le personnage comme l'a ressenti Ezra lors de sa relation avec Hilda dans La Fortune du passager : «Il l'étouffait. L'aimait-il ? Il en était ivre. Des semaines sans femmes, des mois d'esseulement. Et pourtant, c'est avec elle que la solitude l'envahissait. Pour chasser les ombres, le lit, une halte, en

\footnotetext{
${ }^{25}$ Naïm Kattan, La Mémoire et la promesse, p. 8.

${ }^{26}$ Naïm Kattan, L'Autre Amérique dans La Parole et le lieu, p. 100.
} 
attendant un retour de solitude $»^{27}$. De plus, le sexe apparaît comme une solution provisoire, qui ne permet que de mettre en suspens temporairement les problèmes qui reviendront aussitôt la consommation terminée et les effets de cette plénitude temporaire estompés. À l'image des consommateurs de drogues classiques, la personne en souffrance s'enferme dans un cercle vicieux en y ayant recours de façon répétitive et excessive, et ne peut s'en sortir uniquement et véritablement que lorsqu'elle trouve une réponse définitive, qu'elle arrive à régler ses problèmes d'ordre identitaire ou avec son entourage. Ni apaisant, ni salvateur, le sexe utilisé sous cette forme peut même avoir une influence négative sur le comportement d'une personne en la rendant effrayante ; son besoin de combler à tout prix ce vide, de trouver un(e) partenaire permettant de briser cette solitude, de l'aider à faire face à ses problèmes peut faire naître un effet de répulsion, une forme de dégoût chez le partenaire.

Cette approche de la sexualité débridée liée à une situation d'exil chez Naïm Kattan se retrouve aussi chez certains écrivains juifs aux parcours personnels similaires à celui de l'intellectuel irako-canadien comme Eli Amir, Shimon Ballas ou bien Sami Michael comme le montre Nancy E. Berg dans son essai Exile from exile - Israeli writers from $\operatorname{Iraq}^{28}$. Cette dernière va encore plus loin dans son analyse en associant sexe et exil avec le personnage de l'adolescent. En effet, la position dans laquelle se retrouve l'adolescent est la même que celle que vit l'exilé : tous les deux sont dans une période transitoire dominée par les interrogations, les remises en question personnelles auxquelles ils doivent faire face. Leur monde originel s'effondre, leurs convictions, leurs valeurs sont ébranlées, bousculées. Tous les deux se cherchent une place dans ce nouveau monde dans lequel ils ont été jetés bien malgré eux, et c'est au cours de cette période de remise en question que l'adolescent, tout comme l'exilé, construit sa nouvelle identité à travers les différents choix qu'il effectue. Dans l'univers kattanien, tout comme dans les romans des auteurs sur lesquels Nancy E. Berg s'est penchée, la très grande majorité des personnages en souffrance sont principalement de jeunes adultes ou des personnages (masculin ou féminin) dans la force de l'âge. Nous n'avons pas affaire à des personnages qui sont sur les derniers instants de leur vie.

\footnotetext{
${ }^{27}$ Naïm Kattan, La Fortune du passager, p. 280.

${ }^{28}$ Nancy E. Berg, Exile from exile - Israeli writers from Iraq, New-York, State University of New-York press, 1996, 156 p., anglais.
} 
Les relations hommes-femmes peuvent parfois revêtir un caractère fusionnel, passionnel et, dans l'univers kattanien, ce sont majoritairement les femmes qui partent dans cet extrême où elles sont tellement cantonnées qu'elles finissent généralement par faire fuir leur partenaire, comme Dina dans La Célébration ou bien Nina dans la nouvelle Nuit de tempête dans le recueil Le Sable de l'île ${ }^{29}$. Ces femmes, pourtant fortes en apparence, souvent bien en place au niveau professionnel et/ou familial, sont en réalité d'une grande fragilité, et recherchent à travers cet amour fusionnel un réconfort, quelqu'un sur qui s'appuyer comme pour Dina : « J'ai cru que tu étais parti pour de bon. Après un moment de colère, ça a été le désespoir. Je me voyais : une épave. Le vide total. Tu m'avais abandonnée, tu ne voulais plus de moi. Pour surnager, je me serais accrochée à n'importe quoi $\gg{ }^{30}$. Ce besoin de combler un vide s'explique aussi par le fait que Dina se sent délaissée par un mari absent mais, en se penchant sur le passé de la jeune femme, on comprend également qu'elle a été contrainte d'épouser son mari pour faire plaisir à des parents ultraconservateurs, dominateurs, et n'a décidé de se marier que pour fuir cet autoritarisme parental ${ }^{31}$. D'un point de vue psychologique, l'attitude de la jeune femme peut également être interprétée comme une réponse à une "déficience narcissique »: Houari Maïdi justifie cette attitude de la jeune femme, en s'appuyant sur les théories freudiennes, et en affirmant que les personnes qui ont subi, ou pensent avoir subi, tant de souffrance s'estiment en droit de s'excepter de toute loi ou morale et recherchent le plaisir proche et immédiat: "Ces sujets estiment être victimes innocentes d'un patrimoine héréditaire inique et légitimisent la faute de leurs actes, puisqu'elles ont enduré la violence de leurs ascendants $»^{32}$.

Si ce sont surtout des femmes qui sont à la recherche du grand amour, les hommes sont également à la recherche de la femme idéale qui leur permettra de (re)trouver une certaine sérénité. Cette recherche éperdue rappelle également une certaine idée de l'unité originelle perdue entre l'homme et la femme que l'on retrouve dans de nombreuses mythologies. Dans la mythologie grecque, on considérait l'être humain comme étant à l'origine hermaphrodite, l'homme et la femme ne formant qu'un et vivant en harmonie. Et c'est suite à une décision divine que les

\footnotetext{
${ }^{29}$ Naïm Kattan, Le Sable de l'île, Paris, Gallimard, 1981, 177 p.

${ }^{30}$ Naïm Kattan, La Célébration, p. 100.

${ }^{31}$ Naïm Kattan, La Célébration, pp. 61-62.

${ }^{32}$ Houari Maïdi, Narcissisme et clinique anthropologique à l'adolescence dans Marieke Wolf-Fedida, Bilinguisme, interculturel et ethnologie, concevoir l'autre et l'autrui, MJW Fédition, 2013, 144 p.
} 
deux sexes furent séparés. On retrouve cette même conception de la création de l'homme et de la femme dans le judaïsme comme le montre le professeur Howard Schwartz dans son livre Tree of Souls: The Mythology of Judaism: The Mythology of Judaism ${ }^{33}$. Deux versets bibliques sont à l'origine de cette hypothèse : «Dieu créa l'homme à son image ; c'est à l'image de Dieu qu'il le créa. Mâle et femelle furent créés à la fois » ${ }^{34}$ et « Il les créa mâle et femelle, les bénit et les appela l'homme, le jour de leur création ${ }^{35}$. C'est deux versets ont donné lieu à deux interprétations distinctes sur cet épisode de la création d'Adam: la première sous-entend une double création (Adam ET Eve). En revanche, une deuxième interprétation que l'on retrouve dans le Midrash Rabbah tend à faire penser que Dieu créa à l'origine un être hermaphrodite, duel (physique et spirituel) avant de les séparer en deux êtres distincts ${ }^{36}$. La recherche du grand amour apparaît dès lors comme une volonté, de la part d'un individu, de retrouver à travers l'autre sexe cette unité originelle perdue. L'homme a par conséquent besoin de la femme et, inversement, pour se retrouver, pour être pleinement lui/elle.

Le besoin mutuel de l'autre sexe, la recherche éperdue de l'unité originelle perdue c'est aussi, comme l'affirme Claude Dubar dans La Crise des identités, l'interprétation d'une mutation $^{37}$, la possibilité pour cet individu de se (re)découvrir :

La rencontre de l'être aimé, écrit justement Roussel, « installe les amoureux dans un nouveau monde », un monde à part, enchanté, merveilleux. L'être aimé apparaît d'une richesse inépuisable, singulière, irréductible. C'est une révélation « comme si chacun trouvait enfin sa propre identité dans le même temps où il découvre celle de l'autre. C'est une véritable renaissance, une transfiguration comme si chacun était, pour l'autre, toute sa raison de vivre. Les amoureux entrent dans un « ailleurs », un monde nouveau qui leur ferait dire : « Cet univers est une grâce ». Je ne peux plus me passer de lui, je le découvre et, en même temps, je me découvre. Nous sommes comme des miroirs qui nous reflètent l'un l'autre, parfois nous nous y confondons. C'est une cristallisation. Ainsi, l'autre me révèle à moi-même, comme je le révèle à lui-même, de manière nouvelle. (p. 81)

\footnotetext{
${ }^{33}$ Howard Schwartz, Tree of Souls : The Mythology of Judaism : The Mythology of Judaism, Oxford University Press, 2004, 704 p.

${ }^{34}$ Genèse, I, 27.

${ }^{35}$ Genèse, V, 2.

${ }^{36}$ Howard Schwartz, Tree of Souls... op. cit., pp. 138-139.

${ }^{37}$ Claude Dubar, La Crise des identités, l'interprétation d'une mutation, Paris, PUF, 2010, 256 p. Les caractères en italiques et les expressions entre guillemets sont dans le texte cité.
} 
L'Autre, en plus de me permettre de me (re)trouver, me permet de ne pas disparaître, de ne pas m'enfoncer dans l'anonymat comme l'affirme Naïm Kattan ; c'est à travers l'Autre que je vis, mais c'est aussi cet Autre qui me fait vivre, qui me fait sentir vivant ${ }^{38}$.

Selon l'intellectuel irako-canadien, l'homme et la femme sont également l'un pour l'autre, en plus d'être une porte d'accès à une meilleure connaissance d'eux-mêmes, une ouverture sur l'extérieur. En évoquant sa propre expérience, il montre comment, par le biais des différentes femmes qu'il a rencontrées dans sa vie, il a découvert de nouvelles cultures, de nouveaux univers, et a pu ainsi s'enrichir :

D'ailleurs, c'était toujours mêlé, quand je parle des femmes, à la découverte d'une culture. Je parle d'une Polonaise, c'était tout un côté slave que je découvrais. Je parle d'une Hollandaise, c'est toute une culture germanique, hollandaise. La Française dont je parle... où j'allais dans des châteaux, de grandes maisons en province, c'était toujours toute une société que je découvrais. C'était pour quelqu'un qui est l'étranger mais qui ne voulait pas être étranger. C'était quelqu'un qui vient de l'extérieur. Il arrive dans une société et il n'y a qu'un moyen de pénétrer cette société, par son désir, son désir de pénétrer cette société. Or, le chemin le plus court, c'est un circuit d'ordre affectif. On ne peut pas aimer une ville sans aimer ses habitants. Alors, les habitants, pour un jeune homme, ça passe d'abord par le circuit le plus rapide et le plus intense, le plus riche, il faut le dire, c'est celui de la femme. ${ }^{39}$

Excepté le personnage de Farida dans le roman éponyme, on constate que les femmes jouent, la plupart du temps, un rôle secondaire dans l'univers kattanien, que ce soit dans les écrits fictifs ou bien à travers ses essais ou ses articles qui sont essentiellement consacrés à des hommes. Elles ont la plupart du temps des rôles plutôt négatifs et, comme nous venons de le voir, elles sont très souvent décrites comme faibles, abandonnées, désespérées, voire ne servant qu'à dispenser aux hommes un plaisir temporaire. Elles sont généralement plus un moyen, une consolation qu'une finalité en soi permettant au personnage masculin de soulager une souffrance, le soutenir dans sa quête. Le personnage masculin est toujours, comme nous venons de l'affirmer, dans l'action, dans le mouvement, à la recherche de ce petit quelque chose qui lui manque pour parvenir au bonheur. Et il est même prêt à tout abandonner pour y parvenir. Ezra dans La Fortune du passager, Gabriel dans Le

\footnotetext{
${ }^{38}$ Naïm Kattan, La Mémoire et la promesse, p. 158.

39 Jacques Allard, «Entrevue avec Naïm Kattan» dans Voix et images, vol. 11, ${ }^{\circ} 1$, 1985, pp. 10-32.
} 
Gardien de mon frère, Edgar dans Le Réveil des distraits ou Léo dans Le Long retour ${ }^{40}$ parcourent le monde à la recherche du bonheur, la sérénité qu'ils n'ont plus chez eux. Quant à Pierre-Nathan, dans La Célébration, il abandonne tout (emploi, femme, maîtresse) lui aussi pour essayer de se retrouver. Cette quête du bonheur est aussi marquée de tension, d'une certaine rivalité avec les personnages masculins, jouant parfois des coudes entre eux pour parvenir à leurs fins. Encore une fois, seules les femmes parviennent parfois à briser cette tension, à les canaliser comme pour Éliahou dans Le Veilleur qui ne parvient à faire taire son esprit rebelle vis-à-vis de la religion qu'à partir du moment où il fait la connaissance d'Emma, sa future épouse.

On peut affirmer cependant, en lisant les différents entretiens qu'il a eus et dans lesquels il a abordé sa jeunesse, qu'il existe une certaine dichotomie entre la description que donne Naïm Kattan des personnages masculins et féminins par rapport à la considération qu'il a de la gente féminine et de son expérience personnelle avec les hommes qui lui ont été proches. Très jeune, il s'est auto-proclamé défenseur des femmes (et plus généralement des plus faibles) dans une société patriarcale, machiste oppressante à leur égard. Naïm Kattan se sent en désaccord total avec cette mentalité prédominante dans la société irakienne des années 19301940. Il décrit une société dans laquelle les hommes et les femmes ne semblent se rencontrer uniquement à travers le sexe, licite ou non, et qui ne vivent pas ensemble. Ils se croisent chaque jour, mais sans avoir de véritables relations humaines, et même dans le domaine familial. En ce qui concerne ses relations avec le monde masculin, la dichotomie est moins prononcée; on retrouve en effet ces tensions intramasculines, auxquelles nous avons fait référence, dans ses relations difficiles avec son frère aîné ou son meilleur ami Nessim. En revanche, il donne une description de son père aux antipodes du héros kattanien : homme absent, vivant (volontairement?) dans l'ombre des autres membres de la famille, incapable de tenir son rôle de chargé de famille, d'homme de la maison. Cette image d'un père effacé explique en grande partie le manque d'admiration du fils pour ce dernier. On ressent une certaine souffrance du jeune garçon face à ce père soumis aux autres et à la vie. Il trouve même une figure paternelle de substitution en la personne de son oncle maternel Menaché pour lequel il voue une totale admiration ${ }^{41}$.

\footnotetext{
${ }^{40}$ Naïm Kattan, Le Long retour, Montréal, Éditions Hurtubise, 2011, 292 p.

${ }^{41}$ Sophie Jama, Entretiens avec Naïm Kattan, Montréal, Liber, 2005, 243 p., pp. 22-23.
} 


\title{
3 L'Orient et l'Occident, sources bibliques et sources juives
}

L'une des dualités les plus importantes présentes dans la pensée kattanienne, et qui possède un caractère particulier pour l'auteur, est son lien avec l'Orient et la gestion de ce lien avec l'Occident qui fait désormais partie de sa vie :

\begin{abstract}
Toute sa vie l'Irak restera présent dans son œuvre même si cela n'est pas forcément dit. Il suffit de lire Kattan pour retrouver, au détour de quelque phrase, l'ambiance feutrée des maisons closes de Bagdad, la poétique de ses ruelles, les rencontres spontanées dans ses cafés, l'humour caustiques des Bagdadi, la présence permanente d'un Bagdad vécu et rêvé. Oui, l'Irak est toujours au cœur de son œuvre. Oui, Naïm Kattan est irakien pour l'éternité. ${ }^{42}$
\end{abstract}

Naïm Kattan, par son choix de vie, est marqué dans sa chair par cette dualité qui se ressent, comme l'affirme Abdul Kader El Janabi, jusque dans ses écrits ; l'Irak, et plus généralement l'Orient, demeure très présent dans le cœur de Naïm Kattan. Même si l'Amérique, et en particulier le Canada, occupe une place majeure dans l'univers de l'auteur, nombreuses sont les références, explicites ou non, à l'Irak de son enfance ou à l'Orient. Il y a dans l'univers kattanien beaucoup de personnages irakiens ou orientaux comme dans le roman Farida qui se déroule à Bagdad, mais aussi à travers le personnage d'Éliahou dans Le Veilleur ou d'Ezra dans La Fortune du passager qui sont irakiens. Il y a également Mounira (égyptienne d'origine) dans Le Réveil des distraits, Éli et Sarah (respectivement tunisien et égyptienne d'origine) dans L'Amour reconnu, ou encore René Shems dans L'Anniversaire (origine syrienne). L'Orient se retrouve aussi en arrière fond dans certaines nouvelles comme dans Rue Abou Naouas ${ }^{43}$, L'Hôtel $^{44}$ ou Le Visiteur ${ }^{45}$. Il est aussi présent, comme nous l'avons déjà affirmé, de façon plus implicite notamment à travers le Brésil.

Cette présence orientale dans ses différents écrits s'explique du fait que, comme pour un grand nombre de Juifs irakiens, l'Irak préexilique apparaît comme un paradis sur terre. Les Juifs irakiens sont, dans leur très grande majorité, fiers de leur pays, de leur communauté et de tout ce

\footnotetext{
${ }^{42}$ Citation de Abdul Kader El Janabi dans Simone Douek, Naïm Kattan, l'écrivain du passage, (sous la dir. de Jacques Allard), Montréal, Éditions Hurtubise, 2004, pp. 89-90. Abdul Kader El Janabi (né en 1944 à Bagdad) : poète, journaliste et écrivain irakien.

${ }^{43}$ Naïm Kattan, Dans le désert, Montréal, Éditions Leméac, 1974, 153 p.

${ }^{44} \mathrm{Ibid}$

${ }^{45} \mathrm{Ibid}$
} 
que celle-ci a apporté à leur pays. Fiers aussi du patrimoine cultuel que leurs ancêtres ont transmis à l'ensemble de la communauté juive. Même si des événements tragiques comme le Farhud $^{46}$ ou l'expulsion forcée de tous les Juifs de ce pays en 1951 apparaissent comme des tâches sombres, quasi indélébiles (événements qui reviennent plusieurs fois dans différents romans de Naïm Kattan), l'auteur, comme tous ses coreligionnaires, n'a jamais renié ses origines, son patrimoine culturel, cultuel ou linguistique. C'est cet amour de la terre, transmis de génération en génération, que l'on retrouve dans la pensée kattanienne et dans ses différents écrits. Faire transparaître l'Irak dans ses différentes œuvres est une façon de témoigner son amour, rendre hommage à cette terre qui l'a vu naître et sur laquelle il a grandi. Il utilise même ses personnages, comme avec Ezra dans La Fortune du passager, pour faire poindre une certaine nostalgie face à un passé révolu.

Cette attitude de la part de l'intellectuel irako-canadien n'est pas un cas isolé dans la mesure où on la retrouve également chez certains auteurs juifs d'origine irakienne qui ont également connu cette période préexilique et qui se sont installés en Israël, comme chez Samir Naqqash ou Yitzhak Bar Moshe. L'attachement à la terre originelle s'est traduit chez eux par un attachement à la langue arabe, quitte à s'isoler d'un large public, mais aussi par le contenu de leurs ouvrages. Tous les deux se sont en effet concentrés sur la communauté juive d'Irak et les lieux dans lesquels ils vivaient, tentant ainsi de préserver le très riche et ancien héritage culturel des Juifs d'Irak ${ }^{47}$. Même les écrivains qui ont fait le choix pragmatique de l'hébreu comme langue d'expression, tel que Sami Michael ou Shimon Ballas, ont conservé un lien avec leur Irak natal dans leurs œuvres, même si ce n'était pas leur unique source d'inspiration. Sans entrer dans une certaine nostalgie castratrice qui pourrait les empêcher de vivre, continuer d'avancer malgré leur déracinement, ils introduisent dans leurs écrits, tout comme le fait Naïm Kattan, des éléments explicites ou non qui prennent la forme d'une reconnaissance, la traduction d'un amour inébranlable pour leur pays d'origine.

L'Orient se retrouve également à travers le judaïsme qui occupe une place importante dans la vie de notre auteur, une importance qui donne dès lors une touche orientale à son univers. Pour Naïm Kattan, la religion, tout comme la langue arabe, constitue l'un des éléments fondamentaux de son identité. Toute sa vie, il a fait en sorte de maintenir

\footnotetext{
${ }^{46}$ Émeutes sanglantes anti-juives qui se déroulèrent à Bagdad le $1^{\text {er }}$ et le 2 juin 1941.

${ }^{47}$ E. Nancy Berg, Exile from exile... op.cit., p. 53.
} 
un lien fort avec le judaïsme, pour qu'il ne meure pas, refusant toute forme ou toute invitation à l'assimilation ${ }^{48}$. L'intellectuel irako-canadien est fier de sa judéité et même s'il a eu, au cours de sa vie, certains moments de rébellion, il ne pouvait s'empêcher, s'interdire de respecter certains préceptes religieux comme la célébration de Yom Kippour ${ }^{49}$. Un tel lien s'explique aussi par le fait que l'auteur s'est approprié le livre saint par analogie, en s'identifiant à son contenu. Il a fait du récit biblique sa propre histoire : « La Bible constitue ma propre histoire. Lorsque je lis la Bible, c'est de moi qu'il s'agit, quand bien même il y a beaucoup d'autres choses $»^{50}$. Une identification personnelle aux Saintes Écritures qui s'est renforcée avec les similitudes que notre auteur a trouvées entre certains membres de sa famille et des personnages bibliques : son grand-père maternel s'appelait Joseph, et ce dernier a prénommé ses deux fils Menaché et Ephraïm, tout comme Joseph dans le livre de la Genèse $e^{51}$. Il retrouve également en la personne de son oncle maternel Menaché la figure du Joseph de la Bible ${ }^{52}$.

La Bible a aussi beaucoup influencé son style, son travail littéraire ou bien le contenu de ses écrits. En ce qui concerne son style, l'auteur explique que son penchant pour le récit lui vient en grande partie de la Torah et des textes religieux comme le Talmud: «Il y a des histoires incroyables dans le Talmud. C'est un livre de narrations autant que de commentaires. Il y a des essais et il y a des histoires. Et peut-être que d'une manière inconsciente, j'ai hérité cela, sans le savoir ${ }^{53}$. Influence sur son style, mais aussi sur son champ lexical : on retrouve en effet une certaine inspiration biblique dans le choix de titres de ses ouvrages comme le recueil de nouvelles Dans le désert qui fait référence au quatrième livre de la Torah; il l'explique en quatrième de couverture. Naïm Kattan y présente des personnages en attente qui vivent dans un désert et entrevoient leur terre promise. La référence à la traversée du désert par le peuple hébreu ainsi que le champ lexical qui y est associé se retrouve également dans deux autres titres d'ouvrages : La Traversée $e^{54}$ ou Le Long retour. Le titre d'un autre ouvrage de Naïm Kattan semble également trouver son inspiration dans la Bible: Le Gardien de mon

\footnotetext{
${ }^{48}$ Sophie Jama, Entretiens avec Naïm Kattan, op. cit., p. 92.

${ }^{49}$ Ibid, p. 202.

${ }^{50}$ Ibid, p. 11.

51 Ibid.

${ }^{52}$ Ibid, pp. 22-23.

${ }^{53}$ Simone Douek, Naïm Kattan, l'écrivain du passage, op. cit., pp. 39.

${ }^{54}$ Naïm Kattan, La Traversée, Montréal, Éditions Hurtubise, 1976, 152 p.
} 
frère. Il rappelle le verset biblique du livre de la Genèse et qui intervient après le meurtre d'Abel par son frère Caïn : « L'Éternel dit à Caïn : Où est Abel ton frère? Il répondit: Je ne sais; suis-je le gardien de mon frère ? ${ }^{55}$.

Naïm Kattan s'inspire aussi des Écritures Saintes pour écrire. On retrouve parfois des similitudes très prononcées avec certains passages de la Bible, que ce soit à travers le récit, l'intrigue, les personnages ou bien par le message véhiculé (l'exil, l'altérité, ou d'autres que nous n'aborderons pas dans cet article comme la puissance de la parole ou l'universalité). Tout ceci tend à donner aux personnages kattaniens un caractère biblique ne serait-ce, dans une grande majorité, que par la situation personnelle dans laquelle ils se trouvent et leur attitude face à cette situation. Comme nous y avons fait référence précédemment en citant le recueil Dans le désert, ils sont majoritairement dans la même situation psychologique fragile (crise identitaire, amoureuse ou relationnelle, ou bien à la recherche du bonheur) qui les poussent tous à adopter la même attitude : le retrait ou bien la fuite, au sens propre ou bien au sens figuré. Cependant, la fuite n'est pas considérée, dans la pensée kattanienne, comme un acte de lâcheté. Il s'agit plus d'une attitude salutaire pour les personnages dans la mesure où elle leur évite de sombrer dans un mal-être, une tristesse qui deviendrait à long terme insurmontable et qui les isolerait du monde des vivants. La fuite, ou le retrait, s'avère également comme nécessaire dans la mesure où elle permet aux personnages de faire une mise au point sur eux-mêmes, sur leur vie, et par la suite de faire les choix adéquats (s'ils en ont le courage tant ces choix peuvent être parfois difficiles à prendre et/ou à mettre en œuvre). Ce mécanisme psychologique et comportemental chez les personnages kattaniens revêt un caractère religieux, mystique dans la mesure où, dans le judaïsme, tout comme dans de nombreuses religions, la retraite (ou la fuite) permet à l'être élu de pouvoir faire table rase de son passé, de se purifier, de se libérer de tout ce qui le (re)lie à la vie terrestre pour être en mesure de recevoir le message divin. Une purification nécessaire en vue d'accomplir sa mission d'ordre divin.

L'intellectuel irako-canadien s'inspire également de certains personnages bibliques pour ses fictions et c'est certainement le roman $L e$ Gardien de mon frère qui illustre le mieux cette inspiration biblique. En plus du caractère biblique du titre de ce roman, les personnages principaux (Gabriel et Raphaël) apparaissent comme les images quasi-

\footnotetext{
${ }^{55}$ Genèse, IV, 9.
} 
parfaites de deux personnages bibliques : Esaü et Jacob. On retrouve, en effet, dans la figure des deux frères bibliques de fortes ressemblances avec les frères du roman de Naïm Kattan :

- Gabriel / Esaü : tous deux aînés de leur fratrie, ils représentent tous les deux l'image du chasseur, l'homme de la terre, le guerrier ${ }^{56}$. Ils sont tous les deux des hommes à femmes ${ }^{57}$.

- Raphaël / Jacob : les deux cadets de leur famille respective sont, à l'inverse de leurs frères, beaucoup plus calmes, posés. De plus, comme Jacob, à qui on ne connaît que deux épouses officielles (Léa et Rachel), Raphaël ne fait référence qu'à deux jeunes femmes quand il aborde le sujet de ses relations privées (Sophie et Irène).

Les similitudes ne s'arrêtent pas aux caractéristiques propres à chaque personnage; leurs histoires sont également quasi identiques. La paracha Toledot du livre de la Genèse décrit la rivalité entre les deux frères bibliques qui prend forme dès leur conception, et qui perdure tout au long de leur vie comme Dieu le révèle à Rebecca, la mère des deux enfants $^{58}$. Les relations entre les différents membres de la famille de Raphaël et Gabriel ne sont pas non plus sans nous rappeler celles de la famille biblique, même si Naïm Kattan prend une certaine liberté avec le récit originel en les restructurant. Mais on a cependant toujours affaire à une construction binomiale: Isaac/Esaü (l'aîné) et Rebecca/Jacob (le cadet) d'un côté, et Gabriel (l'aîné)/sa mère et Raphaël (le cadet)/son père de l'autre. On peut enfin ajouter que, tout comme les deux frères bibliques, Gabriel et Raphaël font fi des rivalités qui les animent (ou les animaient) pour se retrouver afin de rendre un dernier hommage à leur père mourant avant de l'enterrer.

Le personnage de Job transparaît également à travers certains personnages de l'univers kattanien. Dans la pensée juive, Job représente la patience, la soumission et le respect inconditionnels à Dieu, et ceci quelles que soient les épreuves, les blessures ou les trahisons. On rencontre dans l'univers kattanien certains personnages qui rappellent, par leur façon de penser ou leurs attitudes, cette importante figure du judaïsme. Dans Le Long retour, Léo fait la rencontre de Julia. La jeune femme apprend à ce dernier qu'avant leur rencontre elle avait connu le grand amour en la personne d'Edwin, l'homme qu'elle avait aimé et qui était mort dans un accident de voiture. Cette tragédie fut doublement

\footnotetext{
${ }^{56}$ Genèse, XXV, 27.

${ }^{57}$ Genèse, XXVIII, 9.

${ }^{58}$ Genèse, XXV, 22-23.
} 
douloureuse pour la jeune femme dans la mesure où c'est à ce moment précis qu'elle apprit l'existence d'une maîtresse qui était reconnue aux yeux des autorités et des gens comme étant la femme officielle. Mais, malgré cette double blessure teintée de trahison, la jeune femme n'a cependant pas cessé de vivre, et c'est avec la musique qu'elle a trouvé un certain réconfort qui lui a permis de reprendre confiance en elle et de croire à nouveau en l'amour : "Elle vivait la musique à sa mesure, ne se sentait pas frustrée de ne pas occuper les premiers rangs. Cette disposition lui avait ouvert les portes de l'amour. Son corps avait repris toute sa dimension et l'habitant, elle le possédait et pouvait en faire don $»^{59}$.

On peut également voir transparaître la figure de Job dans le personnage d'Ezra dans La Fortune du passager. Ce dernier a tout perdu suite à une escroquerie et il a passé une grande partie de sa vie à pourchasser l'escroc en question; une poursuite qui l'a empêché de se poser et de fonder une famille. Il n'a même pas pu être présent pour soutenir et accompagner sa mère lors des derniers instants de sa vie, ni même assister à son enterrement. Mais tout comme Dieu rendra à Job ce qu'il avait perdu, tout sera rendu à la fin à Ezra : il a retrouvé sa fortune et s'est même enrichi grâce aux affaires qu'il a réalisées tout au long de sa vie. Il a également retrouvé une certaine sérénité, perdue depuis des années, et surtout l'amour dans les bras de Tamar (la fille de Jouri Fettal, l'homme qui l'avait escroqué). Comme le résume Naïm Kattan au cours de ses entretiens avec Simone Douek, « il a perdu une fortune, mais il en a gagné une autre ${ }^{60}$. Ezra et Julia qui, après de nombreuses épreuves, ont fait preuve de patience et n'ont jamais cessé de croire, n'ont jamais baissé les bras face à l'adversité. Et le destin a fini par leur rendre ce qu'ils avaient perdu, en mieux, confirmant l'enseignement transmis par la Bible à travers le personnage de Job que l'on peut résumer en reprenant le proverbe : « Tout vient à point à qui sait attendre ».

Les Juifs, le judaïsme et le monde juif dans son ensemble occupent donc une place centrale tant dans la vie de Naïm Kattan que dans son travail et son univers littéraire ; il faut ajouter que, peu de temps après son arrivée au Canada, il a fondé Le Bulletin du cercle juif qui avait pour vocation principale de faire connaître aux Canadiens francophones les Juifs de langue française. Il mit également en place à l'université du Québec à Montréal deux cours en lien avec la Torah: Les figures

\footnotetext{
${ }^{59}$ Kattan Naïm, Le Long retour, p. 243.

${ }^{60}$ Simone Douek, Nä̈m Kattan, L'écrivain du passage, op. cit., pp. 50-51.
} 
bibliques dans la Bible et le Coran, et les mythes contemporains, et Poésie et prophétisme ${ }^{61}$. Du premier cours cité, un ouvrage fut même publié : Figures bibliques : des patriarches aux prophètes ${ }^{62}$.

Ce souci de présenter à tous ce qu'est véritablement la communauté juive et le judaïsme apparaît comme une nécessité pour l'auteur avec pour but de rapprocher ce qui est aujourd'hui éloigné, d'effacer les incompréhensions et les malentendus, de mettre fin aux fantasmes et aux stéréotypes entre cet Orient et cet Occident qu'il connaît bien. Il existe une réelle fracture entre l'Orient et l'Occident, fracture qui remonte à l'époque antique comme l'illustrent les guerres que ont opposé la Grèce et la Perse. Depuis cette période, les deux mondes n'ont eu de cesse de s'affronter sans véritablement chercher à se comprendre, se respecter, apprendre l'un de l'autre. Une relation ambiguë, à la limite de la paranoïa, faite d'un mélange, de la part des deux camps, d'attraction-rejet alternant le rapprochement et l'éloignement, s'est installée ; une attitude que Naïm Kattan aborde dans certains de ses écrits. On retrouve, par exemple en filigrane dans la pensée kattanienne, l'image de l'Européen rebelle, agité, destructeur qui reprend cette perception orientale ancienne de l'Occidental, image qui s'affirme notamment à travers le courant romantique au cours du $\mathrm{XIX}^{\mathrm{e}}$ siècle. Dans son essai Le Réel et le théâtral, Naïm Kattan décrit le personnage de l'adolescent romantique ; il le définit comme étant " refus et affirmation », " présence et évasion », un personnage qui apparaît en totale opposition avec l'adolescent de la période classique ${ }^{63}$. L'Occidental, tout comme le personnage de l'adolescent romantique du $\mathrm{XIX}^{\mathrm{e}}$ siècle, est un rebelle, un individu qui remet en cause les règles préétablies, qui rejette le monde tel qu'il est tout en refusant d'y prendre part. On trouve, dans cette description faite par Naïm Kattan de l'adolescent romantique, de nombreux traits caractéristiques chez certains de ses personnages, et notamment chez le personnage de Gabriel dans Le Gardien de mon frère : il y a chez le jeune homme une instabilité (amoureuse, géographique, professionnelle), le tout mélangé à un esprit de rébellion qui en fait l'incarnation de l'Occident. Par opposition, son frère Raphaël qui est beaucoup plus calme, posé, respectueux de ses aînés, apparaît donc comme le pendant oriental. La relation qu'entretiennent les deux frères devient de facto une

\footnotetext{
${ }^{61}$ Sophie Jama, Entretiens avec Naïm Kattan, op. cit., p. 131.

${ }^{62}$ Naïm Kattan, Figures bibliques : des patriarches aux prophètes, Montréal, Éditions Guerin, 1997, 169 p.

${ }^{63}$ Naïm Kattan, Le réel et le théâtral, p. 21.
} 
illustration des relations entre l'Orient et l'Occident, une relation alternant le chaud et le froid, dans laquelle se retrouvent des sentiments aussi divers qu'antagonistes : la rivalité, le respect, l'amour, la colère ...

Il n'y a cependant pas, dans la conception kattanienne, de rejet de l'Occident, ou un quelconque soutien à cette réponse violente que l'Orient peut donner par moments. La réponse doit et ne peut se trouver que dans le dialogue, l'échange mutuel. C'est une démarche que l'intellectuel irako-canadien promeut comme en témoigne sa tentative de rapprocher les communautés juives anglophone et francophone, ou bien les communautés littéraires canadiennes francophones et anglophones. Une démarche reconnue et appréciée par certains de ses pairs : " Il faut savoir reconnaître dans ces actions la parole d'un écrivain prêt à unir le monde des idées dans la réconciliation des cultures ${ }^{64}$. Naïm Kattan rejette et se refuse à tout retranchement, tout enfermement hermétique au monde extérieur, qu'il soit assumé ou subi. Ce rejet se traduit notamment dans son écriture et dans ce refus de se voir enfermé dans un cadre stéréotypé, refusant de jouer le rôle de l'écrivain exotique, l'autre venu d'ailleurs. Il a donc, selon ses dires, fait en sorte d'occidentaliser ses écrits, et estime y être parvenu en ayant recours à la narration qui s'appuie plus sur l'histoire que sur le mot, mais aussi en abandonnant toutes fioritures, emphase, débordement de style qui caractérisent l'écriture orientale ${ }^{65}$. Bien évidemment, cette symbiose ne doit pas être synonyme d'assimilation qui serait, selon l'auteur, comme une mort de l'identité de la personne ${ }^{66}$. Naïm Kattan est conscient de la difficulté de vivre en symbiose avec différentes cultures. Ceci explique son admiration pour ceux qui y parviennent comme cet Irakien assyrien travaillant à la représentation française à Bagdad qu'il rencontra et auquel il fait référence dans Adieu Babylone. Ce dernier avait réussi « la difficile union entre ses origines et son pays d'adoption $»{ }^{67}$.

Le dialogue, qui n'a jamais été véritablement présent au cours de l'histoire des relations occidento-orientales, semble être la seule solution pour parvenir à faire taire les discours de haine et abattre les murs d'incompréhension. Seuls des intellectuels comme Naïm Kattan qui ont fait le choix, volontaire ou non, de se confronter, de s'ouvrir à ces

\footnotetext{
${ }^{64}$ Citation du poète et écrivain canadien Jean Royer (né en 1938) dans Simone Douek, Naïm Kattan, l'écrivain du passage, op. cit., p. 101.

${ }^{65}$ Sophie Jama, Entretiens avec Naïm Kattan, op. cit., pp. 143-145.

${ }^{66} \mathrm{Ibid}, \mathrm{p} .92$.

${ }^{67}$ Naïm Kattan, Adieu Babylone, p. 299.
} 
différents mondes, en mettant en avant le meilleur aux détriments du moins bon, permettent, par la force de leur témoignage et de leurs écrits, de créer ce dialogue constructif et positif. De plus, au-delà d'une paix retrouvée, cette ouverture d'esprit entre les deux entités sont également les bases pour favoriser un enrichissement tant matériel qu'intellectuel, l'un pouvant apporter à l'autre ce dont il a besoin, ce qui lui manque, et inversement. Comme l'explique Georges Corm, dans son essai OrientOccident, la fracture imaginaire ${ }^{68}$, l'Histoire a montré que sans une certaine ouverture à l'autre, en particulier à l'Orient qu'il a longtemps combattu, l'Occident n'aurait jamais pu atteindre un tel rayonnement culturel :

Que serait la Grèce ancienne s'il n'y avait pas eu de contacts intenses avec la civilisation égyptienne et les grandes civilisations d'Asie Mineure, celle des Perses et des Mèdes et celles des grands empires mésopotamiens ? C'est la contribution de toutes ces populations qui conduira la grande civilisation hellénique, dominant l'Orient (jusqu'aux invasions arabes) aux côtés de la culture syriaque, elle-même héritière des civilisations babylonienne, chaldéenne, araméenne, assyrienne. Et c'est la civilisation musulmane, on l'oublie trop souvent, qui protégea ce patrimoine et le transmettra à l'Europe, lui permettant ainsi de bâtir sa renaissance intellectuelle sur lui. ${ }^{69}$

Selon Naïm Kattan, l'Orient est plus qu'un vecteur culturel pour l'Occident, il fait partie intégrante d'une grand partie de la culture européenne :

La civilisation occidentale est une intégration malaisée jamais terminée de deux pôles : un christianisme venu d'Orient et une mythologie grecque proposant non pas uniquement une explication du monde mais son organisation. C'est la civilisation grecque puis romaine qui ont prévalu mais l'Orient a toujours eu une présence en Occident, triomphante ou persécutée, victorieuse, ou réduite à la dissimulation. Elle fut et est toujours là. ${ }^{70}$

C'est l'Orient qui a contribué à faire de l'Occident ce qu'il est aujourd'hui, et il se trouve encore présent en filigrane dans l'identité européenne comme par exemple dans la musique :

\footnotetext{
${ }^{68}$ Georges Corm, Orient-Occident, la fracture imaginaire, Paris, La découverte, 2004, $208 \mathrm{p}$.

${ }^{69}$ Ibid, p. 51.

70 Naïm Kattan, Écrivains des Amériques Tome 3 - L'Amérique latine, Montréal, éditions Hurtubise, 1980, 165 p.
} 
Bach est un musicien d'église, un musicien de la religion. Et la musique religieuse est passée de l'Orient vers Byzance, c'est-à-dire vers la Grèce. Quand je suis allé en Grèce écouter de la musique dans les églises, c'était une musique plus ou moins empruntée à l'Orient. Ensuite cette musique est allée vers l'autre Occident. À mon avis, la musique religieuse prend lointainement sa source dans la musique juive et arabe. Et je retrouve cela dans Bach. ${ }^{71}$

Aujourd'hui, en réponse à « l'agression occidentale », c'est l'Orient qui s'enferme dans une attitude, une logique de repli sur lui-même similaire à celle de l'Europe moyenâgeuse. En témoigne le triomphe du fondamentalisme musulman et la montée d'un obscurantisme qui prédominent dans le monde arabo-musulman. Mais l'Orient semble également oublier que la civilisation dont il est issu a pu rayonner dans de nombreux domaines durant des siècles grâce notamment à de multiples apports extérieurs (perse, grec, byzantin, indien etc.); une civilisation qui, pour y parvenir, a admis, intégré également en son sein des communautés ethniques et religieuses diverses. Non seulement, cette attitude ne permet pas de contredire les stéréotypes négatifs à son encontre de la part de l'Occident, mais elle empêche également toute possibilité à l'Orient de redevenir un moteur culturel comme il le fut autrefois $^{72}$.

\section{Une illustration de l'obsession de la dualité chez Kattan : Farida}

Farida raconte l'histoire d'une jeune juive irakienne animée par le goût pour la chanson, qui souhaite faire de la musique son métier, et qui fait tout pour parvenir à ses rêves. Pour y arriver, elle s'appuie sur Salim, un Juif qui a réussi dans les affaires et qui est, aux dires de la jeune femme, l'homme de sa vie, même si les deux amants ne se sont pas mariés officiellement. Elle peut également compter sur Jawad son amant, musulman et chef de la police de Bagdad, qui lui assure, par ses fonctions, protection et confort matériel. L'Histoire, la cupidité humaine, mais également une certaine fierté personnelle d'être parvenue à son objectif, lui font perdre ses deux amants; les deux hommes prennent la fuite pour échapper à la prison à vie, ou bien à une mort plus ou moins certaine. Ce roman, qui a reçu le prix Athanase David ${ }^{73}$ en 2004, est un

\footnotetext{
${ }^{71}$ Sophie Jama, Entretiens avec Nä̈m Kattan, op. cit., p. 181.

${ }^{72}$ Georges Corm, Orient-Occident, la fracture imaginaire, op. cit., pp. 130 -131.

73 Prix Athanase David: créé en 1968 sous le nom de Prix David, il récompense l'ensemble de la carrière et l'œuvre d'un écrivain québecois.
} 
exemple intéressant pour illustrer l'obsession de la dualité dans la pensée kattanienne, une dualité que l'on retrouve en effet sous de nombreuses formes.

Bien que l'action se déroule uniquement à Bagdad, on constate que l'Europe reste présente par l'influence du Royaume-Uni sur le pays, mais aussi par l'évocation de la poussée du nazisme en Allemagne qui se répand progressivement dans le pays en réponse à la montée du sionisme. La situation décrite dans le roman montre aussi comment, par sa présence et son attitude, l'Occident, s'immisçant de plus en plus dans les affaires intérieures du pays, impose ses valeurs, son mode de vie et de penser, et en vient à déstabiliser cet Orient dans lequel la vie suivait paisiblement son cours.

Naïm Kattan pousse la "dualité géographique », réelle à travers la dualité Orient-Occident, plus loin encore à travers la dualité Karradah Sulaykh. Les deux quartiers de Bagdad sont, en effet, les deux lieux de résidence de Farida, et chacun d'entre eux représente des valeurs, des mondes différents voire parfois antagonistes (juif/musulman, simple citoyen de «second zone »/symbole du pouvoir et de l'armée, la famille et la simplicité/le luxe et l'extravagance). Une dualité géographique liée à la dualité amoureuse avec d'un côté Salim le Juif qui demeure à jamais, comme nous l'avons dit, l'homme de sa vie, et à qui elle dédie toutes ses chansons, et de l'autre, Jawad le Musulman pour qui son amour apparaît sincère mais «secondaire» (p. 136). Ce double amour ne semble d'ailleurs absolument pas gêner la jeune femme pour qui la situation n'apparaît pas incohérente. Elle estime, en effet, être capable d'aimer ces deux hommes à la fois (pp. 135-136).

Dualité géographique, mais aussi temporelle que l'on retrouve sous la forme de fractures temporelles dans le roman avec l'utilisation de flash-back. Même si elles sont moins nombreuses et moins flagrantes que dans d'autres romans, l'auteur remonte parfois brutalement dans le temps au cours du récit. Par exemple, lors de la visite de Naji à Farida à la demande de Salim qui est emprisonné pour le meurtre de Sasson, le chapitre suivant revient quelques mois en arrière sur la demande en mariage du père du défunt de la main de Najiah, sœur de Salim et future épouse de Sasson (pp. 71-79).

La dualité présent-passé se trouve également dans ce roman par le fait que le récit se situe dans une période transitoire, une période charnière, ce qui sous-entend qu'il y a un passé qui ne sera bientôt plus, et un avenir en gestation rempli d'incertitudes et d'inquiétudes. Salim, l'un 
des personnages principaux du roman ressent ce virage qu'est en train de prendre le pays (p. 219).

Autre élément caractéristique de la littérature kattanienne présente dans ce roman et empreinte de dualité, la rivalité entre les deux frères Salim et de Naji, ce dernier demeurant le préféré de la famille (pp. 9-10).

La seule exception à tout ce que nous venons de voir, et nous y avons fait référence, c'est que le personnage central du roman reste le personnage féminin. Farida sait ce qu'elle veut et personne ne doit se mettre sur sa route; elle n'hésite pas à menacer sa tante de l'entraîner dans la honte en laissant sous-entendre qu'elle va se fourvoyer dans la prostitution si elle ne la laissait pas prendre des cours de musique (p. 97). Même Salim ne doit pas et ne parvient pas à l'empêcher de réaliser son rêve de chanter (pp. 87-88). Une attitude qui vient même à rendre Salim ridicule, pathétique (p. 92). Elle se moque des conventions, des traditions ou des mœurs (pp. 83 et 85). Même si Farida apparaît comme un personnage fort, elle n'en demeure pas moins, finalement, un personnage solitaire, un personnage seul contre tous. D'un côté, il y a la jeune femme, et de l'autre Salim, Jawad, sa famille et tous les autres (p. 217).

La dualité occupe tellement l'univers kattanien, est tant exploitée et mise en avant sous toutes ses formes, explicite ou non, par l'auteur qu'on peut dire qu'elle apparaît comme l'une des "marques de fabrique » de l'intellectuel irako-canadien. Une telle démarche stylistique, que l'on peut qualifier de quasi obsessionnelle peut s'expliquer par le fait que la dualité apparaît comme étant d'une certaine façon inscrite dans les gènes de l'auteur; Naïm Kattan a toujours vécu dans un univers où régnait la dualité. Comme il l'a toujours ressenti, que ce soit dans son Irak natal ou encore à son arrivée au Canada, la relation à l'Autre est très majoritairement pris sous la forme d'une confrontation, d'une rivalité, ou bien d'un combat en vue de protéger une culture, une langue ou une identité. Cependant, contrairement aux peurs que peuvent susciter l'altérité, Naïm Kattan tend plus à considérer l'Autre (et donc la dualité) comme un bienfait, un atout pour l'homme. En effet, si ce dernier accepte cet Autre de façon positive et non comme une menace, il pourra dès lors mieux se comprendre, mieux se connaître lui-même, mieux s'accepter, et ainsi (re)trouver une sérénité. Mais au-delà d'une meilleure acceptation de soi, cette nouvelle relation avec l'Autre lui permettra également de s'ouvrir de nouveaux horizons, sources d'enrichissement. Pour le bienêtre et le bonheur de l'être humain, tant au niveau individuel qu'à un 
niveau supérieur, l'Autre doit être considéré comme complémentaire, tout comme l'homme et la femme se complètent, et non comme un danger pour l'unité d'un individu ou d'un groupe. De plus, cette nouvelle position vers laquelle nous devons tendre apparaît, d'un point de vue éthique, mais également religieux, comme une obligation pour chacun d'entre nous, une obligation face à laquelle nul ne peut se défiler, et ceci même si la présence de l'Autre peut faire poindre, comme nous venons de l'affirmer, un semblant de menace. 